\title{
Moodle como ambiente MOOC: orientações para o redesign de interação
}

\author{
Beatriz Brito do Rêgo - IME/UFBA - beatrizbr@ufba.br Filipe \\ Adeodato Garrido - IME/UFBA - filipe.garrido@ufba.br \\ Ecivaldo de Souza Matos - IME/UFBA - ecivaldo@ufba.br
}

\begin{abstract}
Resumo: A universidade é um ambiente de construção de conhecimento científico, criada para atender as necessidades da sociedade. Nesse sentido, ambientes de cursos abertos online em massa, como os MOOC (Massive Open Online Courses), são opções adequadas para esse propósito. Ambientes de MOOC ampliam as possibilidades de democratização da educação, por sua dimensão livre e aberta. Nesse sentido, este artigo apresenta os resultados iniciais de avaliação do Moodle para sua adequação como plataforma MOOC, do ponto de vista dos mecanismos de avaliação da interação humano-computador. Acredita-se que a customização do Moodle reduziria os custos de implementação de uma plataforma de MOOC, considerando as especificidades, características, identidade das instituições e sua ampla adoção no Brasil. Por meio da aplicação do Método de Inspeção Semiótica, com os resultados da avaliação indicaram orientações para o redesign de interface do Moodle de modo que contemple elementos necessários a uma plataforma de MOOC.
\end{abstract}

Palavras-chave: MOOC; Interação Humano-Computador; Moodle.

\section{Moodle as a MOOC environment: guidelines for interaction redesign}

Abstract: The university is a local of scientific knowledge construction. It was created to attend the needs of society. In this sense, open online courses environments, such as MOOC (Massive Open Online Courses), are appropriate options for this purpose. MOOC environments expand the possibilities of democratization of education, due to its free and open dimension. In this sense, this paper presents the initial results of an evaluation of Moodle for its suitability as a MOOC platform, from the point of view of the human-computer interaction evaluation mechanisms. It is believed that customization of Moodle would reduce the costs of implementing a MOOC platform, considering the specificities, characteristics, identity of the institutions, and their wide adoption in Brazil. Through the application of the Semiotic Inspection Method, the results of the evaluation indicated guidelines for the Moodle interface redesign so that it contemplates elements necessary to a MOOC platform.

Keywords: MOOC; Human-Computer Interaction; Moodle.

\section{Introdução}

As universidades são culturalmente ambientes de construção e difusão de conhecimento acadêmico, profissional e científico (Vieira e Vieira, 2003; Takahashi e Amorim, 2008). Nesse sentido, ambientes de cursos abertos online em massa (Massive Open Online Course - MOOC) são opções para difusão do conhecimento desenvolvido na universidade, bem como possibilidade de formação continuada de profissionais.

Os MOOC, por definição, possuem como principais características ser aberto, conteúdo acessível e livre de custos para que qualquer pessoa; participativo, a 
aprendizagem dos estudantes baseia-se na criação e no compartilhamento das contribuições pessoais; distribuído, as atividades devem ocorrer em ambientes onde os estudantes interajam fisicamente distantes; e escalável, ou seja, não possuir quantidade predefinida de participantes (Baturay, 2015).

Porém, a viabilidade econômica dos MOOC ainda é um assunto que divide opiniões, por causa do alto custo para desenvolver e manter uma plataforma MOOC (Bastos e Biagiotti, 2014).

Algumas universidades brasileiras utilizam MOOC como apoio de ensino. Uma delas é a Universidade Federal do Rio Grande do Sul (UFRGS) que lançou a plataforma Lúmina ${ }^{1}$, com seu conteúdo desenvolvidos pelos professores universitários, estudantes e técnicos da UFRGS. Outra iniciativa é o Veduca ${ }^{2}$, que disponibiliza o material criado por meio de parcerias com universidades estrangeiras e brasileiras.

Atualmente, o Moodle ${ }^{3}$ (Modular Object-Oriented Dynamic Learning Environment) é um dos ambientes virtuais de aprendizagem mais utilizados para suporte a cursos a distância e presenciais nas universidades federais (Sabbatini, 2007). Concebido como uma plataforma (software) livre, ele possui uma comunidade de desenvolvedores e contribuintes tecnológicos que tem criado plugins para as mais diversas necessidades (Dougiamas e Taylor, 2003).

Software livre é um software cujo código fonte é aberto a modificações por qualquer usuário que queira ou necessite adaptá-lo (Damasio e Ribeiro, 2006). Essa flexibilidade proporcionada pelo software livre, permite com que seus usuários tenham alguma liberdade sobre o sistema (Campos, 2006).

Questiona-se, portanto, se haveria possibilidade de assunção do Moodle como uma plataforma de MOOC, reduzindo os custos de implementação de uma plataforma de MOOC institucional. Para isso, o Moodle precisaria se adequar às características definidoras de um MOOC já citadas.

Nesse sentido, este artigo apresenta resultados da avaliação do Moodle para sua adequação ao uso como MOOC, do ponto de vista dos seus mecanismos de interação humano-computador. Por meio da aplicação do Método de Inspeção Semiótica, avaliamos se o Moodle apresenta elementos de interação que dificultem ou favoreçam seu uso como plataforma de MOOC. Ao final, são apresentadas algumas orientações para o redesign de interação do Moodle, de modo que ele possa atender às exigências mínimas de adaptação a uma plataforma de MOOC.

O texto deste artigo está agrupado em cinco seções. Na próxima seção serão apresentados aspectos conceituais sobre MOOC e Interação Humano-Computador, como foco no conceito de metacomunicação. Em seguida, descrevemos o percurso da inspeção executada na seção sobre a metodologia. Na seção sobre os resultados, são apresentados dados sobre a inspeção realizada, complementada por críticas quanto a adequação do Moodle a uma plataforma de MOOC. Por fim, nas considerações finais, deixamos algumas orientações para o redesign de interface do Moodle para ser usado como plataforma de MOOC.

\section{MOOC e a metacomunicação}

\footnotetext{
${ }^{1}$ https://lumina.ufrgs.br/

${ }^{2}$ https://veduca.org/

${ }^{3}$ https://www.moodle.org
} 
Segundo Grainger (2013), o termo Massive Open Online Course (MOOC) foi criado no ano de 2008 por Dave Cormier para descrever um determinado modelo de curso online desenvolvido por Stephen Downes e George Siemens. O curso em questão era apoiado em princípios conectivistas, ou seja, considerava que o conhecimento é distribuído através de uma rede de conexões, na qual a aprendizagem consiste na capacidade de construir e percorrer essas redes (Downes, 2012).

Conforme Baturay (2015), os MOOC possuem quatro características-padrão: abertos, participativos, distribuídos e escaláveis. Uma das principais iniciativas no desenvolvimento dos cursos MOOC foi a criação de plataformas tecnológicas específicas para suporte a cursos com essas características (Grainger, 2013). Algumas plataformas são conhecidas mundialmente, como o Coursera ${ }^{4}, \mathrm{EDx}^{5}$ e Udacity ${ }^{6}$.

Há pelo menos duas categorias fundamentais de MOOC, com base em diferentes ênfases pedagógicas e modelos organizacionais: cMOOC e xMOOC (Grainger, 2013). Os cMOOC baseiam-se na abordagem pedagógica conectivista, fundamentado nas conexões e no compartilhamento de conteúdo entre os estudantes (Mota e Inamorato, 2012). Os xMOOC se caracterizam pelo uso dos materiais multimídia, principalmente vídeos, quiz e fóruns para interação e avaliação dos estudantes.

Por ser um ambiente educacional online, a interação entre os sujeitos é fundamental. Logo, os aspectos de interação humano-computador e de design pedagógico (ou design instrucional) são fundamentais. Um desses aspectos é a comunicabilidade.

Sob o ponto de vista da Engenharia Semiótica, a comunicabilidade é o potencial interacionista da plataforma tecnológica.. Engenharia Semiótica é uma teoria que compreende a interação (humano-computador) como um processo de comunicação sujeitos (projetistas e usuário da tecnologia) por meio da metacomunicação de interface (De Souza, 2005).

A metacomunicação é o "diálogo" entre os projetistas e os sujeitos-usuário sobre as possibilidades, funcionalidades e restrições interacionistas de determinada tecnologia interativa, como os softwares (Salgado e De Souza, 2007).

Há atualmente duas ferramentas metodológicas da Engenharia Semiótica para avaliação do potencial de comunicabilidade de um ambiente de software: Método de Inspeção Semiótica (MIS) e Método de Avaliação da Comunicabilidade (MAC) (De Souza, 2005; De Souza e Leitão, 2009).

Nessa perspectiva, para interação adequada aos objetivos do design educacional ou de interação faz-se necessário haver elementos que facilitem a compreensão da metamensagem dos projetistas pelos sujeitos-usuário, por meio de signos de interface; evitando rupturas de (meta)comunicação.

As rupturas de comunicação interferem diretamente na qualidade da comunicabilidade em plataformas de MOOC. Em um estudo preliminar, Do Rêgo et al. (2017) correlacionaram rupturas de comunicabilidade às evidências de causas gerais de abandono em cursos MOOC. Os autores levantaram indícios de que a desistência ou abandono de cursos MOOC pelos estudantes se deve também a aspectos de comunicabilidade.

\footnotetext{
${ }^{4}$ https://www.coursera.org/

${ }^{5}$ https://www.edx.org/

${ }^{6}$ https://br.udacity.com
} 


\section{Metodologia}

O Moodle é um ambiente virtual de aprendizagem desenvolvido com o intuito de ser compatível, flexível e de fácil modificação/atualização, permitindo a configuração e customização da interface e dos serviços pelas instituições em instalações próprias (Dougiamas e Taylor, 2003).

Ainda que tenha características tão importantes para o uso como ambiente virtual de aprendizagem institucional, acredita-se que há outras características intrínsecas à qualidade da comunicabilidade que podem comprometer a assunção do Moodle como MOOC institucional. Para se tornar uma plataforma de MOOC, além do gerenciamento do ensino e da aprendizagem dos estudantes, é necessário promover participação intrínseca, distribuição e fundamentalmente abertura, ou seja, todos devem poder se inscrever em seus cursos.

Nesse estudo, focalizou-se apenas os aspectos relacionados à participação e abertura do Moodle para configuração como ambiente MOOC, ou seja, aspectos relacionados diretamente à qualidade da interação humano-computador. Portanto, analisou-se a possibilidade de adequação dos elementos de interação do Moodle, considerando os seus potenciais de comunicabilidade, por meio do MIS.

Por meio do MIS, o avaliador procura por rupturas da comunicabilidade na emissão da mensagem de metacomunicação dos projetistas, sem a participação direta dos sujeitos-usuário (De Souza e Leitão, 2009).

Esse método possibilita que os avaliadores reconstruam a intenção dos projetistas ao criar cada função do sistema. Isso possibilitaria identificar as funcionalidades existentes no software, bem como suas limitações.

Metodologicamente, o estudo foi dividido nas três fases compositoras do MIS: (i) inspeção dos signos de interação; (ii) reconstrução da metamensagem de cada conjunto de signos; e (iii) consolidação dos resultados.

Na primeira fase, inspeção dos signos de interação, houve análise dos três conjuntos de signos, conforme a Engenharia Semiótica: metalinguísticos, estáticos e dinâmicos. Os signos metalinguísticos são usados pelos projetistas para comunicar explicitamente aos sujeitos-usuário os significados atribuídos aos demais signos codificados na interface e como eles devem ser usados (Bim e De Souza, 2010). Os signos estáticos expressam o status do ambiente, cujo significado deve ser interpretado não obstante as relações causais e temporais da interface. Por sua vez, os signos dinâmicos expressam o comportamento do sistema, envolvendo aspectos temporais e causais da interface (Barbosa e Silva, 2010).

$\mathrm{Na}$ segunda fase houve a elaboração da mensagem de metacomunicação correspondente de cada uma das três classes de signos. A terceira fase consistiu na consolidação dos resultados, em que o avaliador sumarizou as rupturas encontradas na plataforma, identificando os elementos sígnicos referente aos conhecimentos prévios necessários e impostos pela plataforma para navegação pela sua interface; além dos conhecimentos adquiridos após a navegação/inspeção do sistema. Após a sumarização das rupturas encontras, os avaliadores propuseram sugestões de melhorias para a interface, em conformidade com as orientações de Barbosa e Silva (2010).

A avaliação ocorreu entre janeiro e maio de 2018, sobre a implementação do Moodle institucional da Universidade Federal da Bahia (UFBA). Todavia, limitações 
técnicas podem interferir na adaptação para um MOOC. Atualmente, são permitidos até 80 acessos simultâneos no Moodle-UFBA.

\section{Resultados}

Realizada por dois estudantes de pós-graduação com a supervisão de um especialista em IHC, a inspeção e identificação dos signos de interface ocorreu de forma autoguiada, seguindo esta ordem: inspeção dos signos metalinguísticos, estáticos e dinâmicos. Os resultados estão descritos na ordem de organização dos signos.

Na página inicial há um box "Informações básicas" e expressões que denotam textos de ajuda para o usuário. Há um texto em que o projetista fala diretamente ao usuário, informa que o acesso completo ao sistema requer uma conta. $\mathrm{O}$ espaço Acessar é para o usuário que deseja acessar aos cursos do Moodle, mediante autenticação ${ }^{7}$. Situado ao lado direito do botão "Acessar", há o link "Criar uma conta", sem destaque quando comparado ao botão "Acessar" ( $c f$. Figura 1). Desse modo, entendemos que os projetistas não incentivam a criação de contas por requisição direta do usuário, provavelmente pelo uso frequente do Moodle para apoiar atividades presenciais.

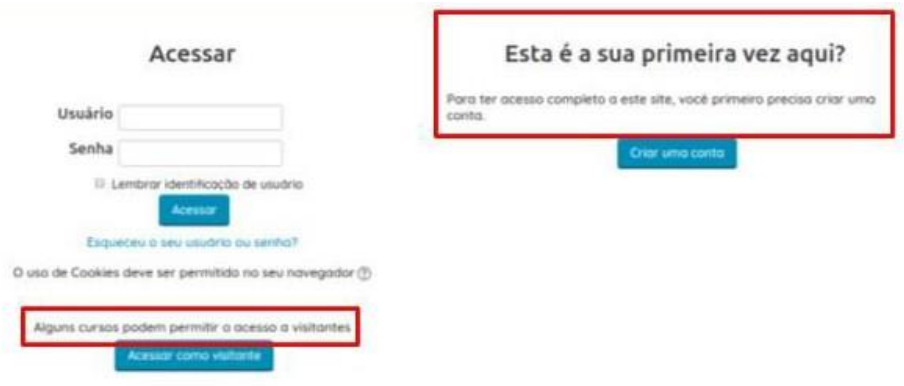

Figura 1 - Página de acesso ao Moodle

Para o sujeito-usuário autenticado na plataforma, sem experiência no Moodle, está disponível no box "Sobre o Moodle", o guia de funcionalidades Moodle 2.6 ( $c f$. Figura 2), localizado na página inicial. Em cursos MOOC não há necessariamente tutores; portanto, os papéis disponibilizados pela plataforma são suficientes para a criação de um MOOC até esse momento da inspeção.

Não há central de ajuda no Moodle avaliado, elemento fundamental em qualquer plataforma de MOOC.

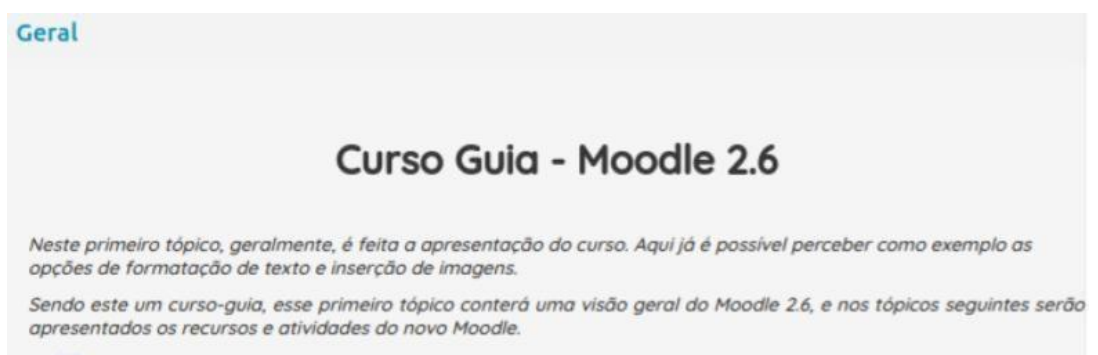

Figura 2 - Página principal curso guia

Algumas funções do Moodle possuem tooltips ${ }^{8}$ com descrição do funcionamento

\footnotetext{
${ }^{7}$ Autenticação é o processo de verificação de usuário e senha no servidor.

8 Janela pop-up que apresenta-se quando passa-se o mouse sobre um elemento da interface, as mesmas contém uma explicação adicional sobre tal elemento.
} 
de determinado signo da interface. Uma dessas tooltips informa que o checkbox posicionado ao lado direito de cada tarefa, ao ser selecionado, representa que a coleção vídeos foi assistida (concluída). Porém, nota-se que algumas informações estão incompletas, evidenciado pelo uso de reticências ao final da informação apresentada (ver Figura 3).

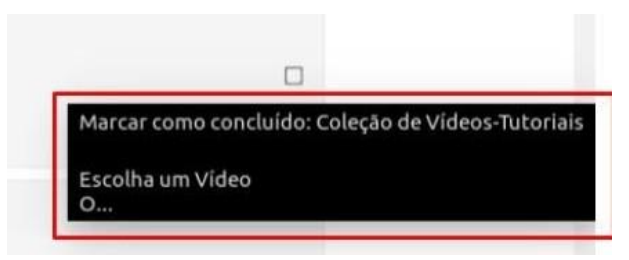

Figura 3 - Tooltip do quadrado de conclusão de atividade

No menu Informações básicas (usuário autenticado), há um submenu Orientações Moodle. Esse submenu pode ser comparado à central de ajuda de outros ambientes ou plataformas. Nele há a informação de que o Moodle é personalizado para a instituição, pois, além do brasão há o nome da universidade no topo da página.

Nesse submenu também é explicado como cada classe de usuário (professores, estudantes, público externo) pode utilizar o Moodle. Ao final do texto, o projetista avisa ao usuário que em caso de dúvida ele deverá fazer contato por e-mail. Isso substituiu a lista de perguntas frequentes $\left(\mathrm{FAQ}^{9}\right)$ verificada em inspeção anterior (em janeiro de 2018). Atualmente a seção perguntas frequentes foi retirada desse menu, prejudicando usuários que por ventura tenham dúvidas básicas (gerais) na utilização da plataforma.

Para o usuário-moderador, a opção Adicionar uma atividade ou recurso (função inexistente para o usuário-estudante), disponível em qualquer curso que o usuário exerça esse papel, apresenta uma janela com as atividades e recursos disponíveis para inclusão. Ao lado dos itens disponíveis para adição, há o seguinte informativo "Selecione uma atividade ou recurso para exibir a sua ajuda. Dê um duplo clique no nome de uma atividade ou recurso para adicioná-lo rapidamente." A mensagem é clara e explicativa.

A atividade "GeoGebra" exibe o texto explicativo em inglês, diferente da maioria das atividades e recursos disponíveis, igual as atividades BigBlueButtonBN, Checkmark, Jogo - (Forca, Milionário, Sopa de Letras e Palavras-cruzadas) e Diário. Isso ressalta a característica monolinguística da plataforma.

No momento em que o usuário-moderador seleciona a opção tarefa, na janela "Adicionar uma nova atividade ou recurso", ele será redirecionado à página "adicionando um(a) novo(a) tarefa". O título da tarefa expressa uma confusão por parte do projetista, pois a frase adicionando um novo tarefa possui erro de concordância. Possivelmente resultado de problemas de tradução ( $c f$. Figura 5).

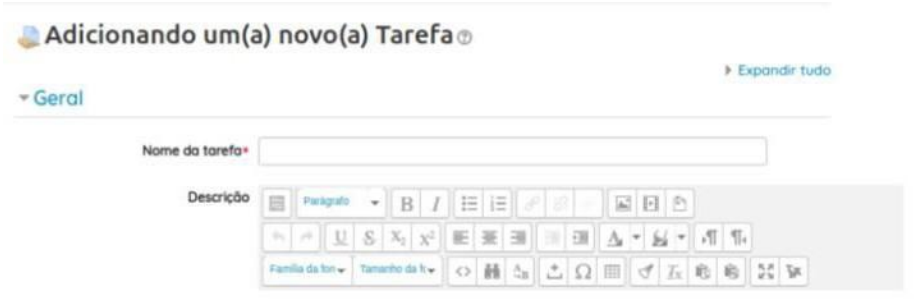

Figura 5 - Página adicionar uma nova tarefa

\footnotetext{
${ }^{9}$ Termo originalmente em inglês, frequently asked questions (perguntas mais frequentes) 
A informação obrigatória para criação de tarefa é o campo nome da tarefa (título); a sua descrição não é obrigatória. Pelo fato de o preenchimento desse campo não ser obrigatório, entende-se que o Moodle é uma ambiente de apoio ao ensino presencial, quando a tarefa pode ser descrita ao(s) estudante(s) em sala. Porém, como plataforma de MOOC, carece de modificações, como a obrigatoriedade de descrição da tarefa.

Cada tarefa criada possui um ícone para sua representação, sendo eles os ícones para PDF, para arquivo de imagem, para arquivo de vídeo, para pasta e para livro. $\mathrm{O}$ arquivo de extensão PDF (Portable Document Format) utiliza a logomarca da Adobe Acrobat Reader $^{\odot}$, leitor de PDF amplamente conhecido; arquivos de imagem utilizam uma câmera fotográfica como representação do mundo físico. Arquivos de vídeo são identificados por um símbolo que se assemelha a um relógio, acompanhado do texto “Arquivos Windows Media Player”, cuja associação semiótica não é imediata.

Ao responder um tópico no fórum somente o campo de mensagem é obrigatório. Ao final da página há dois botões: Enviar mensagem ao fórum e Cancelar. Notou-se que o botão Enviar mensagem ao fórum possui destaque em comparação ao botão Cancelar, este está levemente sombreado. Isso potencialmente induz o usuário a clicar no botão destacado ( $c f$. Figura 6).

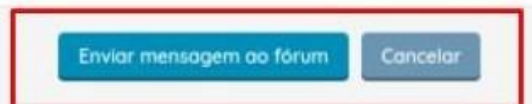

Figura 6 - Botões de envio de mensagem ao fórum e de cancelamento desse envio

No curso cujo papel do usuário é o de moderador, um botão intitulado Ativar edição está disponível no topo da página. Como o próprio nome do botão sugere, permite ao usuário editar conteúdo(s) (recursos/tarefas) do curso. No botão utiliza-se uma imagem de caderno e lápis. Isso indica possibilidade de criação, mas não de edição. Porém, ambas são ações disponíveis ao moderador. Funções fundamentais no gerenciamento de uma plataforma de MOOC.

Ao ativar a edição, automaticamente o botão se modifica para Desativar edição, como também o símbolo, substituído por um símbolo de ligar e desligar. Similar ao encontrado em controles de aparelhos domésticos, dissonante do botão Ativar edição. Após a ativação da edição, alguns elementos novos são apresentados na interface, como as opções de "editar e adicionar uma atividade ou recurso".

Existe a representação de uma Checklist para que o usuário-estudante possa marcar a atividade como concluída, caso essa marcação não seja automática. Ao clicar sobre o a forma geométrica retangular, o Moodle informa dinamicamente ao usuário com o símbolo, semelhante ao utilizado para carregamento em outros sistemas, que ele deve aguardar até o ciclo ser completado para preencher o quadrado ( $c f$. Figura 7).

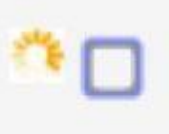

Figura 7 - Imagem para indicar conclusão de atividade ou tarefa

O menu Meus cursos apresenta apenas a lista dos cursos em que o sujeito está inscrito, seja como estudante ou moderador. Ao passar o mouse sobre o menu, a lista aparece instantaneamente, ocasionando confusão para o usuário que imagina a interação 
por meio do clique no menu, sendo direcionado a outra página com uma lista detalhada dos cursos e com informações detalhadas de cada curso. Para a criação de um MOOC será necessário modificar a apresentação dos cursos. Os estudantes deverão visualizar todos os cursos disponíveis na plataforma, não apenas os cursos que estão vinculados.

$\mathrm{Na}$ instância de Moodle avaliado há uma barra de progresso (opcional) para que os estudantes possam acompanhar seu rendimento no curso. À medida em que o estudante marca a realização das tarefas no checklist, essa barra é atualizada. A barra de progresso é representada pelas cores vermelho e verde, em que vermelho representa as atividades que não foram realizadas e verde as que foram marcadas como concluídas pelo estudante (ver Figura 8).

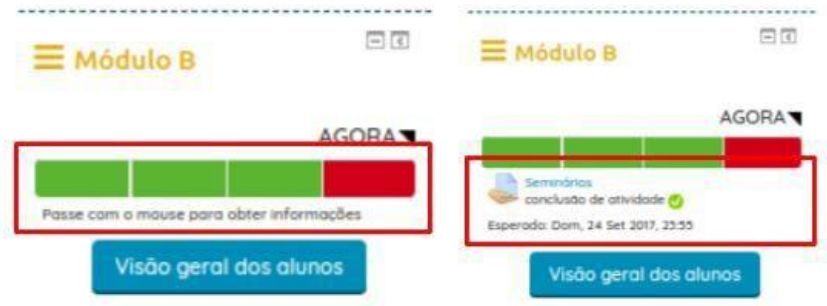

Figura 8 - Barra de progresso

Ao passar o cursor do mouse sobre cada quadrado (vermelho ou verde), apresenta-se dinamicamente, abaixo da barra, qual atividade/tarefa está sendo representada. Esse detalhamento pode ser útil ao usuário para verificar quais tarefas estão pendentes. Caso o usuário clique sobre a tarefa, será automaticamente direcionado à tela da atividade/tarefa, reduzindo o tempo de busca.

\section{Considerações Finais}

Neste artigo foi apresentada a avaliação da qualidade da metacomunicação de uma instância de Moodle, por meio de aplicação do MIS. As rupturas de comunicabilidade encontradas e descritas geraram três metamensagens reconstruídas sob a perspectiva dos signos metalinguísticos, estáticos e dinâmicos, apresentados na interface. Essa inspeção verificou a redundância, distribuição e (in)consistência das mensagens presentes nos signos.

Concluiu-se, portanto, que o Moodle-UFBA possui interface tão simples e de fácil compreensão, quanto possível, pela utilização de linguagem inteligível e símbolos do mundo físico. Entretanto, notam-se erros primários na representação da metamensagem; erros que, no entendimento dos avaliadores, foram ocasionados por tratar-se de um software concebido de forma colaborativa por uma comunidade virtual que contém diversos projetistas em todo o mundo.

Devido à pluralidade de perfis e necessidades pedagógicas dos cursos MOOC, foram identificados signos de interface não-triviais que precisam ser substituídos ou removidos na customização do Moodle para MOOC. Na instância avaliada, não há uma seção de perguntas frequentes (FAQ), fundamentais em plataformas MOOC, dado que o alto número de sujeitos-usuário inviabiliza atendimento personalizado para dúvidas técnicas específicas sobre o uso do ambiente. Logo, um ambiente de ajuda online é fundamental, mas ausente na instância de Moodle avaliada. Em termos metodológicos, isso prejudicou a avaliação dos signos metalinguísticos, aqueles que expressam os significados dos demais signos, geralmente expressos por meio de FAQ ou sistema de ajuda. 
Ainda assim, acreditamos que o Moodle, considerando a versão avaliada, é uma plataforma que poderá servir como base para um MOOC. Por meio desse estudo, foi possível tecer orientações para sua customização com o propósito de servir como uma plataforma de MOOC. Apresentamos a seguir algumas orientações para essa customização, a saber:

- a plataforma deve ter uma central de ajuda ou FAQ, elemento fundamental em plataformas de uso em massa;

- deve-se permitir acesso à plataforma por usuários externos à instituição;

- não se deve exigir cadastro às pessoas que desejam usar (ou conhecer) os cursos;

- o design instrucional dos cursos deve ser direcionado ao ensino a distância, em vez de apoio ao ensino presencial;

- deve-se possibilitar que todos possa ter acesso aos materiais submetidos pelos demais estudantes, de modo a favorecer a construção colaborativa de conhecimento.

Por fim, espera-se que este artigo contribua na caracterização da metacomunicação do sistema Moodle, para que futuramente as rupturas de comunicabilidade sejam corrigidas ou mitigadas e não propagadas ao (re)modelar a plataforma para dar suporte a um MOOC.

Vale lembrar que as instalações do Moodle podem variar quanto ao design de interface e posicionamento ou ocultamente de alguns elementos de interação, ou até mesmo plugins e funcionalidades disponíveis. Todavia, ainda que em locais distintos, os elementos fundamentais são semelhantes entre as instalações do Moodle. Entretanto, isso não permite a generalização per si dos resultados desse estudo, mas indicações de ajustes necessários em uma instância do Moodle, que podem ser mais ou menos similares a outras instalações do Moodle institucionalmente.

\section{Agradecimentos}

Agradecemos aos membros do Grupo de Pesquisa e Extensão em Informática, Educação e Sociedade - Onda Digital e à Fundação de Amparo à Pesquisa do Estado da Bahia (FAPESB) pelo financiamento desta pesquisa.

\section{Referências}

BARBOSA, S.; SILVA, B. Interação humano-computador. Elsevier. 2010. 383 p.

BASTOS, R. C.; BIAGIOTTI, B. MOOCs: uma alternativa para a democratização do ensino. RENOTE - Revista Novas tecnologias na Educação, Porto Alegre, v.12, n.1, p. 65-80, 2014.

BATURAY, M. H. An overview of the world of MOOCs. Procedia-Social and Behavioral Sciences, v.174, p.427-433, 2015.

BIM, S. A. Obstáculos ao ensino dos métodos de avaliação da engenharia semiótica. Rio de Janeiro: Departamento de Informática/PUC-Rio, 2009. 180p. Tese de Doutorado.

CAMPOS, A. O que é software livre. BR-Linux. Florianópolis, março de 2006.

Disponível em <http://br-linux.org/linux/faq-softwarelivre>. Acesso em: 20 de maio 2018. 
DAMASIO, E.; RIBEIRO, C. Software livre para bibliotecas, sua importância e utilização: o caso Gnuteca. Rev. Digit. Bibliotecon. e Cienc. Inf. / RDBCI, Campinas, v.4, n.1, p.70-86, 2006.

DE SOUZA, C. S. The semiotic engineering of human-computer interaction. Massachusetts (EUA): MIT Press. 2005. 312 p.

DE SOUZA, C. S.; LEITÃO, C. F. Semiotic engineering methods for scientific research in HCI. California (USA): Morgan \& Claypool, 2009. 122 p.

DO RÊGO, B. B.; GARRIDO, F.; MATOS, E. Identifying influences of the quality of interaction on dropout rates of mooc: preliminary results. In: Simpósio Brasileiro de Informática na Educação, v. 28, n. 1, 2017. Recife. Anais. Recife: Sociedade Brasileira de Computação, 2017, p. 1766-1768.

DOUGIAMAS, M.; TAYLOR, P. Moodle: Using learning communities to create an open source course management system. In: EdMedia: World Conference on Educational Media and Technology, 2003, Honolulu/Hawaii (USA). Anais. Waynesville: Association for the Advancement of Computing in Education (AACE). 2003. p. 171-178.

DOWNES, S. Connectivism and Connective Knowledge. Essays on meaning and learning networks. 2012. Disponível em:

<https://www.downes.ca/files/books/Connective_Knowledge-19May2012.pdf> Acesso em 22 mai. 2018.

GRAINGER, B. Introduction to moocs: avalanche, illusion or augmentation. Policy Brief- UNESCO (july). Institute for Information Technologies in Education. 2013. Disponível em: <http://iite.unesco.org/pics/publications/en/files/3214722.pdf > Acesso em 03 abr. 2018.

MOTA, R.; INMORATO, A. Mooc, uma revolução em curso. Jornal da ciência, São Paulo, nov. 2012. Disponível em:

$\langle$ http://www.jornaldaciencia.org.br/Detalhe.jsp?id=85111 > . Acesso em 20 mai. 2018.

SABBATINI, R. M. Ambiente de ensino e aprendizagem via internet: a plataforma Moodle. 2007. Instituto EduMed, v.25, 2007. Disponível em:

$<$ http://www.ead.edumed.org.br/file.php/1/PlataformaMoodle.pdf > Acesso em 03 abr. 2018.

SALGADO, L. C.; DE SOUZA, C. S. Commest-uma ferramenta de apoio ao método de avaliação de comunicabilidade. In: III Conferência Latino-Americana de Interação Humano-Computador (CLIHC). 2007. Rio de Janeiro. Anais. Sociedade Brasileira de Computação. s/p.

TAKAHASHI, A. R. W.; AMORIM, W. A. C. Reformulação e expansão dos cursos superiores de tecnologia no Brasil: as dificuldades da retomada da educação profissional. Ensaio: Avaliação e Políticas Públicas em Educação, Rio de Janeiro, v.16, n.59, p.207-228, 2008.

VIEIRA, E. F.; VIEIRA, M. M. F. Estrutura organizacional e gestão do desempenho nas universidades federais brasileiras. Revista de Administração Pública, Rio de Janeiro v.37, n.4, p.899-920, 2003. 